\title{
Riqueza, composición de la comunidad y similitud de las especies bentónicas de la Subclase Opisthobranchia (Mollusca: Gastropoda) en cinco sitios del litoral de Acapulco, México
}

Species richness, community composition and similarity of benthic species of the Opisthobranchia Subclass (Mollusca: Gastropoda) at five sites on the coast of Acapulco, Mexico

\author{
Pedro Flores-Rodríguez ${ }^{1 *}$, Rafael Flores-Garza ${ }^{\text {*** }}$, Sergio García-Ibáñez ${ }^{1}$, Arcadio \\ Valdés-González ${ }^{2}$, Berenice Grisel Martínez-Vásquez ${ }^{1}$, Yocelin Mora-Marín ${ }^{1}$, \\ Elías Jonadad González-Sandoval ${ }^{1}$
}

\begin{abstract}
'Facultad de Ecología Marina, Universidad Autónoma de Guerrero, Av. Gran Vía Tropical No. 20, Fraccionamiento Las Playas, C.P. 39390, Acapulco, Guerrero, México. *pfloresrodriguez@yahoo.com, **rfloresgarza@yahoo.com

${ }^{2}$ Facultad de Ciencias Biológicas, Universidad Autónoma de Nuevo León, Cd. Universitaria, San Nicolás de los Garza, N.L., México

Abstract.- Subclass Opisthobranchia in Acapulco was studied. The objectives were to know the species richness, community composition based on the abundances, local spatial arrangement and hierarchical location of the species, also compare the similarity between sites. Sampling was carried out from 2009 to 2012 at 5 sites during the day, at low tide, every two months. On the rocky intertidal zone samplings were carried out along the shoreline and in the subtidal zone, sampling was carried out using snorkel conducting divings to a maximum depth of $4 \mathrm{~m}$. Fifty-nine species were identified, and 5 were identified to genus level. Twenty-eight new records were determined to Acapulco. Nudibranchia has the highest value in the number of families, species richness and abundance. The highest species richness was found in the Dorididae family and the greater abundance in the Facelinidae family. The most abundant species were Phidiana lascrucensis, Elysia diomedea, Pleurobranchus aerolatus, Glossodoris sedna and Polycera alabe. Twenty four percent of the species has wide local distribution, meanwhile $32.8 \%$ was semi restricted and $43.75 \%$ restricted. Were found 19 dominant species, representing $28.78 \%$ of the total species. The greatest similarity in abundance was estimated for Tlacopanocha and Parque de la Reina sites. These sites are characterized by similar features in the low wave intensity and a heterogeneous substrate. The species richness found is the highest reported for the American Pacific. Acapulco list of known species of Subclass Opistobranchia is considerably expands and it contributes to the knowledge of the composition of the community of this Subclass.
\end{abstract}

Key words: Opisthobranch, richness, community composition, similarity, Acapulco

\begin{abstract}
Resumen.- Se estudió la Subclase Opisthobranchia en Acapulco. Los objetivos fueron conocer la riqueza de especies, la composición de la comunidad a partir de las abundancias, disposición espacial local y la ubicación jerárquica de las especies, además comparar la similitud entre sitios. Se muestreó de 2009 a 2012 en 5 sitios, durante el día, en marea baja, bimensualmente. En la zona intermareal rocosa se hicieron muestreos siguiendo la línea de costa y en el submareal se efectuaron inmersiones hasta 4 $\mathrm{m}$ por buceo libre. Se identificaron 59 especies, 5 más identificadas hasta género. Son nuevos registros de distribución para Acapulco 28 especies. El Orden Nudibranchia fue el de mayor cantidad de familias, riqueza de especies y abundancia. La mayor riqueza de especies se encontró en la familia Dorididae y la mayor abundancia en la familia Facelinidae. Las especies más abundantes fueron Phidiana lascrucensis, Elysia diomedea, Pleurobranchus aerolatus, Glossodoris sedna y Polycera alabe. El 24,44\% de las especies presentó disposición espacial local ampliada, el 32,8\% disposición semirestringida y el $43,75 \%$ restringida. Se encontró que 19 especies fueron dominantes y representaron el 28,78\%. Se halló la mayor similitud en abundancia en los sitios Tlacopanocha y Parque de la Reina, que presentan características de intensidad de oleaje bajo y sustratos muy heterogéneos. La riqueza de especies encontrada es de las más altas reportada para el Pacífico Americano. Se amplía considerablemente el listado de especies conocidas para Acapulco y se contribuye al conocimiento de la composición de la comunidad de la Subclase Opisthobranchia.
\end{abstract}

Palabras clave: Opisthobranchia, riqueza de especies, composición, similitud, Acapulco 


\section{INTRODUCCIÓN}

Los organismos que pertenecen a la Subclase Opisthobranchia, se caracterizan por presentar una concha interna poco desarrollada o ausente y por desarrollar un cuerpo con una detorsión variable; donde la cavidad branquial y el ano se colocan en el lado derecho o en la parte posterior del cuerpo. En el ambiente marino, se les conoce como babosas marinas. Es un grupo de organismos frecuentemente asociado a sustratos rocosos o duros y son muy bellos debido a sus formas y colores. La amplia gama de colores brillantes, que presentan, tiene un significado biológico de advertencia a sus posibles depredadores y han desarrollado sustancias químicas, que juegan un papel fundamental en la defensa (Ginsburg \& Paul 2001).

Debido a la escasa importancia comercial que hasta el momento se le ha reconocido a esta subclase y a que pueden deteriorarse con facilidad cuando se manipulan, pocos investigadores en México, han mostrado su interés en el estudio de estos organismos. El mayor conocimiento de moluscos se ha elaborado en especies de reconocida importancia comercial o que tienen una concha visible.

De la literatura sobre la Subclase Opisthobranchia para el Pacífico Americano, la mayor cantidad de reportes analizan aspectos con enfoque en taxonomía y sistemática (Millen \& Gosliner 1985, Bertsch 1995, Wagele \& Willan 2000, Hermosillo \& Valdés 2004, Valdés 2004, Valdés \& AnguloCampillo 2004, Millen 2006, Hermosillo et al. 2006, Hermosillo \& Valdés 2007a, b; Millen \& Hermosillo 2007, Camacho-García \& Gosliner 2008; Hermosillo \& Valdés 2008, Zamora \& Naranjo 2008, Behrens et al. 2009, Carmona et al. 2014, Pola et al. 2014, Goddard \& Valdés 2015, Shipman \& Gosliner 2015), también con listados de especies (Bertsh et al. 1972, Angulo-Campillo 2000, Montoya et al. 2001 ${ }^{1}$, Hermosillo 2004, Hermosillo \& Brehens 2005, Hermosillo \& Camacho-García 2006, Nakamura 2007, Bertsch 2008; Hermosillo \& Gosliner 2008, Castillo 2010, Flores-Rodríguez et al. 2014), otros enfoques son los biogeográficos (Gosliner 1991, Barraza 2009, Hermosillo 2003, Bertsch 2009), reproducción y desarrollo (Goddard 2005, Ávila-Poveda et al. 2014), defensa química (Guido \& Ghiselin 1998, Cimino \& Ghiselin 1999, Ginsburg \& Paul 2001), y aspectos ecológicos (Nybakken 1978, Angulo-Campillo 2003, Hermosillo 2006).

En México, la mayoría de los reportes corresponden a las ecoregiones marinas del Pacífico Sudcaliforniano y del Golfo de California (Wilkinson et al. 2009). En las ecoregiones del Pacífico Transicional Mexicano y Pacífico Centroamericano, son pocos los trabajos de investigación y la mayoría corresponden al estado de Jalisco, son escasos en Colima, Michoacán, Guerrero, Oaxaca y Chiapas.

La revisión de antecedentes indicó que hay grandes vacíos en el conocimiento de la Subclase Opisthobranchia, sobre todo en el Pacífico sur mexicano, por esto, el presente trabajo tuvo como objetivos estudiar en Acapulco la riqueza de especies, conocer la composición de la comunidad a partir de las abundancias, disposición espacial local de las especies y la ubicación jerárquica de las especies en la comunidad, además de comparar similitud entre sitios.

\section{MATERIALES Y MÉTODOS}

\section{Área de ESTUdio}

El estado de Guerrero, forma parte de la Ecorregión Marina de América del Norte número 17, llamada Pacífico Transicional Mexicano y se ubica entre la latitud $16^{\circ} 35^{\prime} 24^{\prime \prime}$ a $17^{\circ} 28^{\prime} 12^{\prime \prime} \mathrm{N}$ y longitud $99^{\circ} 25^{\prime} 12^{\prime \prime}$ a $100^{\circ} 33^{\prime} \mathrm{W}$. La costa de Guerrero se sitúa en la unidad morfotectónica número VIII, frente en la cual se tiene una plataforma continental muy angosta. La línea de costa es paralela a la fosa México Mesoamericana. Se clasifica como costa de colisión continental, donde predominan las costas primarias, formadas por movimientos diastróficos, con fallas y costas de escarpes de falla. No obstante, se dan en menor escala costas secundarias de erosión por olas, promontorios y costas de terrazas elevados cortados por oleaje y costas secundarias por deposición marina, así como playas y ganchos de barrera (Carranza-Edwards et al. 1975). La temperatura superficial del agua es casi constante y fluctúa entre 26 y $28^{\circ} \mathrm{C}$. Las mareas son semidiurnas mixtas (De la Lanza 1991), con poca variación en la amplitud de marea que es $\pm 1 \mathrm{~m}$ (www:// tablademareas.com). El municipio de Acapulco, es parte de la costa del estado de Guerrero y se ubica en la Región Marina Prioritaria número 32, para la conservación y uso de la biodiversidad en México (Arriaga et al. 1998).

En esta investigación el área de estudio incluyó 4 sitios en la bahía de Acapulco, y uno en la bahía de Puerto Marqués, Guerrero (Fig. 1), los cuales variaron en tipo de sustrato y exposición al oleaje. En 4 sitios, se muestreó en sustrato rocoso desde la zona intermareal hasta aproximadamente $4 \mathrm{~m}$ de profundidad. Un sitio de muestreo se ubicó en un sistema de canastas suspendidas en una plataforma fija, anclada aproximadamente a $80 \mathrm{~m}$ de la costa. Los muestreos se llevaron a cabo en marzo 2009 hasta abril 2012 con periodicidad bimensual.

\footnotetext{
${ }^{1}$ Montoya P, E Domínguez, L Roa, A Taborda \& M Grijalba. 2001. Nudibranquios y otros moluscos opistobranquios de la Bahía Gaira, y zonas aledañas a Santa Marta (Colombia). En: Resúmenes del IX Congreso Latinoamericano sobre Ciencias del Mar COLACMAR, San Andrés Isla, Colombia, p. 189. Poster
} 


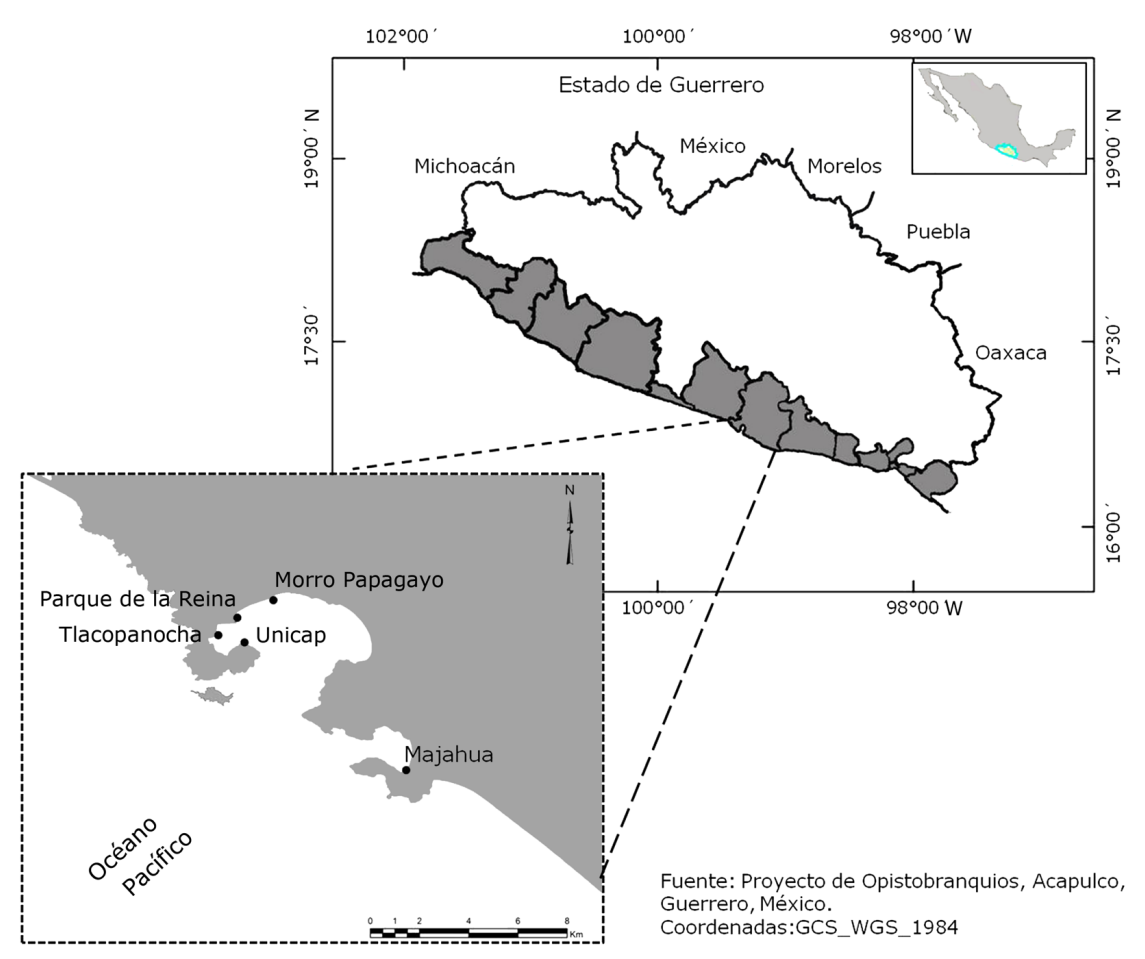

Figura 1. Sitios de muestreo en el litoral de Acapulco, Guerrero, México / Sampling sites on the coast of Acapulco, Guerrero, Mexico

Los sitios ubicados en la zona intermareal tienen diferente grado de exposición al oleaje, dependiendo de esta exposición, los sitios se clasificaron como: a) Alto: cuando las olas chocan directamente en el sustrato, no protegido b) Medio: cuando el impacto de las olas en el sustrato es disminuido por barreras y c) Bajo: cuando las olas no chocan directamente, estos sitios son protegidos por diferentes tipos de barreras.

\section{DESCRIPCIÓN DE LOS SITIOS}

Majahua (16²7’39,62"N y 9950'29,02"W), el intermareal es de acumulación o de aluvión, caracterizada por cantos rodados en procesos de abrasión, cuenta con bloques de textura lisa al tacto y poca presencia de fisuras y/o hendiduras, la intensidad del oleaje es baja; el submareal presenta las mismas características, pero las rocas están pobladas en aproximadamente $80 \%$ de cobertura vegetal y animal, principalmente por especies de algas, briozoarios y esponjas. Hay áreas muy reducidas cubiertas por arena.

Parque de la Reina (1650'58,27"N y 9954’01,85"W), su intermareal es de acumulación artificial de rocas de tamaño variable y consistencia porosa, presenta escombros, restos de piso y pedazos de concreto y la intensidad del oleaje es medio; el submareal con el mismo material pero están recubiertas en aproximadamente el $90 \%$ por varias especies de algas, briozoarios, esponjas, la arena es muy escasa.

Tlacopanocha $\left(16^{\circ} 50^{\prime} 41,53^{\prime \prime} \mathrm{N}\right.$ y $\left.99^{\circ} 54^{\prime} 25,02^{\prime \prime W}\right)$, su intermareal es de acumulación compuestas por rocas de tipo granito-granodiorita y por rocas metamórficas, que varía mucho en cuanto a las dimensiones, ya que es posible encontrar desde grava hasta grandes bloques, la superficie del sustrato es áspero al tacto. La intensidad del oleaje es bajo, el submareal presenta el mismo tipo de material y se encuentran cubiertas por varias especies de algas, briozarios y esponjas, en aproximadamente $60 \%$, la arena es muy escasa.

Papagayo (1651'16,76"N y 9953’05,20"W), es un Islote alejado de la playa a $200 \mathrm{~m}$, su intermareal presenta superficies de arrecifes rocosos cubiertos de algas y esponjas. Al interior se forman pozas de marea que presentan un sustrato arenoso con restos de conchas, y rocas móviles que pueden ser removidas por el impacto de las olas que se clasifica como impacto medio en el lado noreste y bajo en el lado suroeste. El submareal presenta aproximadamente $40 \%$ de recubrimiento de algas en el lado NE y $60 \%$ del lado SW. 
Unicap, es una plataforma flotante ubicada aproximadamente a $80 \mathrm{~m}$ de la orilla dentro de la bahía $\left(16^{\circ} 49^{\prime} 54\right.$ "N y $100^{\circ} 50^{\prime} 27,30^{\prime \prime}$ ). La intensidad del oleaje es baja. La plataforma es utilizada para sostener un sistema de captación de semillas de ostras perleras. De unas líneas penden módulos formados por canastas de plástico tipo Nestier apiladas, dentro de las canastas se colocaron bolsas con luz de malla $(5 \mathrm{~mm})$ que en su interior tiene como colector conchas de ostión. Estos colectores se sumergen a una profundidad máxima de $8 \mathrm{~m}$. Se realiza una limpieza mensualmente de los colectores, y los Opistobranquios se colectaban entre las conchas (Fig. 1).

\section{Recolectas De MUeStras}

El punto de partida de la muestra fue seleccionado al azar y luego una cuerda de longitud de $30 \mathrm{~m}$ fue colocada en paralelo a la línea de costa en la zona intermareal, siguiendo la cuerda y con amplitud de área de $2 \mathrm{~m}$, se recolectaron los Opistobranquios, examinando grietas, oquedades, rocas, entre algas, esponjas y anémonas, hasta completar $1 \mathrm{~h}$.

En la zona submareal el muestreo fue realizado a través de buceo libre, a profundidad máxima de $4 \mathrm{~m}$ hasta completar un tiempo de $1 \mathrm{~h}$.

Los especímenes recolectados fueron depositados en recipientes conteniendo agua de mar para mantenerlos vivos y en buenas condiciones hasta llegar al laboratorio donde fueron colocados en acuarios de 201 de capacidad acondicionados para simular su medio natural.

\section{Trabajo de laboratorio y aNálisis de datos}

Los especímenes colectados fueron identificados y cuantificados. La adecuada ubicación taxonómica requirió de una fase de confirmación en el laboratorio, mediante el uso de literatura especializada (Keen 1971, Behrens \& Hermosillo 2005), se siguió la nomenclatura propuesta por Hermosillo et al. (2006).

Los especímenes vivos fueron depositados en acuarios para facilitar la identificación de especies y fotografiarlos y posteriormente preservarlos mediante técnicas propuestas por Hermosillo (2006), y finalmente ser incluidos tanto en la Colección Malacológica de las Facultad de Ecología Marina de la Universidad Autónoma de Guerrero y en la Colección Nacional de Moluscos en el Instituto de Biología, Universidad Nacional Autónoma de México.

La composición de la comunidad se analizó utilizando la representación a nivel de orden y familia. Esta representación fue evaluada con base en la riqueza de especies y en la abundancia. La abundancia relativa se midió evaluando el porcentaje de cada orden, familia o especie que tienen con respecto al total de especímenes analizados.
La disposición espacial local de las especies de Opistobranquios fue considerada a partir de la presenciaausencia de especies (frecuencia de aparición) en cada sitio de acuerdo al siguiente criterio: A) ampliada: cuando la especie fue encontrada en 4 o 5 sitios B) semirestringida: las encontradas en 2 o 3 sitios y C) restringida: especies encontradas en un solo sitio.

La ubicación jerárquica de las especies dentro de la comunidad, se determinó usando el método de correlación de Olmstead-Tukey, representado mediante gráficas de cuadrantes (Sokal \& Rohlf 1969), que tuvo como base el cálculo de 2 estimadores, el primero fue el promedio de la abundancia relativa de todas las especies, expresado en logaritmo de la abundancia +1 y el segundo estimador, fue el promedio del número de muestras en la cual todas las especies están presentes en los sitios (frecuencia de ocurrencia espacial), expresado en porcentaje. Con el cálculo de estos estimadores se analizó gráficamente el porcentaje de la frecuencia de aparición de cada especie contra el porcentaje de la abundancia relativa promedio de cada especie, expresada en el logaritmo de la abundancia +1 . Las especies dominantes fueron aquellas cuyos valores de abundancia y frecuencia de ocurrencia sobrepasan la media aritmética de ambos valores estimados. Las especies constantes fueron aquellas cuyo valor de abundancia no sobrepasan el valor promedio de la abundancia total, pero si sobrepasan el valor promedio estimado para la frecuencia de ocurrencia. Las especies numerosas poco frecuentes se caracterizaron porque su valor de abundancia es mayor al valor promedio estimado para la abundancia y el valor de frecuencia de ocurrencia no rebasa el valor promedio estimado para esta variable. Las especies ocasionales fueron aquellas que el valor de su abundancia y frecuencia de ocurrencia no sobrepasan a la media aritmética estimada para frecuencia de ocurrencia y abundancia.

Para determinar similitud entre los valores de las abundancias de opistobranquios registradas en los sitios de estudio, se realizó un análisis jerárquico o cluster utilizando la técnica de la distancia euclidiana elevada al cuadrado; previamente se aplicó una transformación de la forma $\log _{10}(x+1)$ a los valores de las abundancias, para lograr la homogeneidad de varianzas dada la presencia de valores extremos y sesgo positivo de cada una de las distribuciones.

\section{Resultados}

Se examinaron 3.431 especímenes de Opistobranquios y se identificaron 5 ordenes, 22 familias, 43 géneros y 63 especies. Las especies: Elysia sp. 1, Cadlina sp. 1, Doto sp.3, Dondice sp. 1, fueron descritas según criterio de Hermosillo et al. (2006). De una especie solo se logró la identificación a género (Eubranchus sp.) (Tablas 1 y 2). 
Tabla 1. Riqueza de especies, abundancia, disposición espacial local y ubicación jerárquica de las especies de los ordenes Cephalospidea, Anaspidea, Notaspidea y Sacoglossa en 5 sitios del litoral de Acapulco, México / Species richness, abundance, local spatial arrangement and hierarchical location, of the order: Cephalospidea, Anaspidea, Notaspidea y Sacoglossa at 5 sites on the coast of Acapulco, Mexico

\begin{tabular}{|c|c|c|c|c|c|c|c|}
\hline \multirow{2}{*}{ Familia / Género / Especie } & \multicolumn{5}{|c|}{ Sitios } & \multirow{2}{*}{ Total } & \multirow{2}{*}{$\mathrm{F}$} \\
\hline & A & $\mathrm{B}$ & $\mathrm{C}$ & $\mathrm{D}$ & $\mathrm{E}$ & & \\
\hline \multicolumn{8}{|l|}{ CEPHALOSPIDEA } \\
\hline BULLIDAE & \multicolumn{5}{|c|}{ Abundancia } & & \\
\hline Bulla punctulata $^{2}$ (A. Adams en Sowerby, 1850) & - & - & - & - & 1 & 1 & $\mathrm{R}$ \\
\hline AGLAJIDAE & & & & & & & \\
\hline Navanax aenigmaticus ${ }^{l}$ (Bergh, 1893) & 3 & 4 & 16 & 9 & - & 32 & A \\
\hline total orden & 3 & 4 & 16 & 9 & 1 & 33 & \\
\hline$S$ (riqueza de especies) & 1 & 1 & 1 & 1 & 1 & & \\
\hline \multicolumn{8}{|l|}{ ANASPIDEA } \\
\hline APLYSIIDAE & \multicolumn{5}{|c|}{ Abundancia } & & \\
\hline Aplysia californica $^{2}$ Cooper, 1865 & 8 & - & 3 & - & - & 11 & SR \\
\hline Aplysia cedrosensis ${ }^{4}$ Bartsch \& Rehder, 1939 & 42 & - & 2 & - & - & 44 & SR \\
\hline Aplysia juliana $^{4}$ Quoy \& Gaimard, 1832 & 85 & - & 8 & - & - & 93 & SR \\
\hline Aplysia parvula ${ }^{l}$ Winkler, 1955 & 21 & 45 & 5 & - & - & 71 & SR \\
\hline Aplysia vaccaria ${ }^{2}$ Winkler, 1955 & 1 & - & - & - & - & 1 & $\mathrm{R}$ \\
\hline Stylocheilus striatus $^{l}$ (Quoy \& Gaimard, 1824). & 5 & 116 & - & 2 & 2 & 125 & A \\
\hline Total orden & 162 & 161 & 18 & 2 & 2 & 345 & \\
\hline$S$ (riqueza de especies) & 6 & 2 & 4 & 1 & 1 & & \\
\hline \multicolumn{8}{|l|}{ NOTASPIDEA } \\
\hline PLEUROBRANCHIDAE & \multicolumn{5}{|c|}{ Abundancia } & & \\
\hline Berthellina ilisima ${ }^{4}$ (Marcus \& Marcus, 1967) & 4 & - & 8 & - & - & 12 & SR \\
\hline Pleurobranchus aerolatus ${ }^{1}$ (Mörch, 1863) & 14 & 16 & 33 & 7 & 318 & 388 & A \\
\hline ONCHIDORIDIDAE & & & & & & & \\
\hline Hoffmanola hansi $^{4}$ (Marcus \& Marcus, 1967) & - & 53 & - & - & - & 53 & $\mathrm{R}$ \\
\hline Total orden & 18 & 69 & 41 & 7 & 318 & 453 & \\
\hline$S$ (riqueza de especies) & 2 & 2 & 3 & 1 & 1 & & \\
\hline \multicolumn{8}{|l|}{ SACOGLOSSA } \\
\hline OXYNOIDAE & \multicolumn{5}{|c|}{ Abundancia } & & \\
\hline Oxynoe panamensis ${ }^{1}$ Pilsbry \& Olsson, 1943 & - & 27 & 20 & 3 & - & 50 & SR \\
\hline \multicolumn{8}{|l|}{ PLACOBRANCHIDAE } \\
\hline Elysia diomedea ${ }^{1}$ (Bergh, 1894) & 54 & 200 & 132 & 52 & - & 438 & A \\
\hline Elysia sp. $^{2}$ & - & 1 & 1 & - & - & 2 & SR \\
\hline \multicolumn{8}{|l|}{ CALIPHYLLIDAE } \\
\hline Cyerce pavonina ${ }^{2}$ Bergh, 1888 & - & - & - & 1 & - & 1 & $\mathrm{R}$ \\
\hline \multicolumn{8}{|l|}{ CORAMBIDAE } \\
\hline Corambe pacifica $^{2}$ (MacFarland \& O’Donoghue, 1929) & - & - & - & - & 1 & 1 & $\mathrm{R}$ \\
\hline \multicolumn{8}{|l|}{ GONIODORIDIDAE } \\
\hline Ancula lentiginosa $^{2}$ (Farmer en Farmer \& Sloan, 1954) & - & - & - & - & 1 & 1 & $\mathrm{R}$ \\
\hline \multicolumn{8}{|l|}{ POLYCERIDAE } \\
\hline Polycera alabe $^{4}$ Collier \& Farmer, 1964 & - & - & - & - & 186 & 186 & $\mathrm{R}$ \\
\hline Total orden & 54 & 228 & 153 & 56 & 188 & 679 & \\
\hline$S$ (riqueza de especies) & 1 & 2 & 3 & 3 & 3 & & \\
\hline
\end{tabular}

Sitios: $A=$ Tlacopanocha, $B=$ Papagayo, $C=P$. de la Reina, $D=$ Majahua, $E=$ Unicap, $F=$ Disposición espacial local, $\left(\mathrm{A}=\right.$ ampliada, $\mathrm{SR}=$ semirestringida, $\mathrm{R}=$ restringida). Ubicación jerárquica $\left({ }^{1}=\right.$ especies dominantes,${ }^{2}=$ ocasional, ${ }^{3}=$ constantes, ${ }^{4}=$ numerosas poco frecuentes) 
Tabla 2. Riqueza de especies, abundancia, disposición espacial local y ubicación jerárquica de las especies del orden Nudibranchia en 5 sitios del litoral de Acapulco, México / Species richness, abundance, local spatial arrangement, and hierarchical location, of the order Nudibranchia at 5 sites on the coast of Acapulco, Mexico

\begin{tabular}{|c|c|c|c|c|c|c|c|}
\hline \multirow{2}{*}{ Familia / Género / Especie } & \multicolumn{5}{|c|}{ Sitios } & \multirow{2}{*}{ Total } & \multirow{2}{*}{$\mathrm{F}$} \\
\hline & A & $\mathrm{B}$ & $\mathrm{C}$ & $\mathrm{D}$ & $\mathrm{E}$ & & \\
\hline \multicolumn{8}{|l|}{ NUDIBRANCHIA } \\
\hline DORIDIDAE & \multicolumn{5}{|c|}{ Abundancia } & & \\
\hline Atagema alba $^{2}$ (O'Donoghue, 1927) & - & - & - & - & 2 & 2 & $\mathrm{R}$ \\
\hline Baptodoris mimetica $^{2}$ (Gosliner, 1991) & - & - & 1 & - & - & 1 & $\mathrm{R}$ \\
\hline Diaulula aurila ${ }^{4}$ (Marcus \& Marcus, 1967) & - & - & - & - & 12 & 12 & $\mathrm{R}$ \\
\hline Diaulula greeleyi ${ }^{1}$ (MacFarland, 1909) & - & - & 4 & 2 & 15 & 21 & SR \\
\hline Discodoris ketos $^{4}$ (Marcus \& Marcus, 1967) & - & - & 3 & - & 35 & 38 & SR \\
\hline Doris granulosa $^{3}$ (Pease, 1860$)$ & - & 1 & 4 & - & 2 & 7 & SR \\
\hline Doris pickensi $^{2}$ (Marcus \& Marcus, 1967) & - & - & - & - & 1 & 1 & $\mathrm{R}$ \\
\hline Hoplodoris bramale ${ }^{2}$ Fahey \& Gosliner,2003 & - & - & - & - & 4 & 4 & $\mathrm{R}$ \\
\hline Geitodoris heathi $i^{3}$ (MacFarland, 1905) & 1 & - & 1 & - & 2 & 4 & SR \\
\hline Jorunna tempisquensis ${ }^{1}$ Camacho \& Gosliner 2008 & - & 3 & 4 & 4 & 17 & 28 & A \\
\hline Taringa aivica ${ }^{2}$ (Marcus \& Marcus, 1962) & - & - & 1 & - & 8 & 9 & SR \\
\hline Thordisa rubescens ${ }^{2}$ (Behrens \& Henderson, 1981) & - & - & 2 & - & 6 & 8 & $\mathrm{~S}$ \\
\hline \multicolumn{8}{|l|}{ CONUALEVIDAE } \\
\hline Conualevia alba ${ }^{2}$ Collier \& Farmer, 1964 & - & - & 1 & 1 & - & 2 & SR \\
\hline Conualevia marcusi $i^{2}$ Collier \& Farmer, 1964 & - & - & 3 & 6 & - & 9 & SR \\
\hline \multicolumn{8}{|l|}{ CHROMODORIDIDAE } \\
\hline Cadlina flavomaculata $^{2}$ (MacFarland, 1905) & - & - & 2 & - & - & 2 & $\mathrm{R}$ \\
\hline Cadlina luarna $^{2}$ (Marcus \& Marcus, 1967) & - & - & 2 & - & - & 2 & $\mathrm{R}$ \\
\hline Cadlina sparsa $^{2}$ (Ohder, 1921) & - & 3 & - & - & 1 & 4 & SR \\
\hline Cadlina sp. $1^{2}$ & - & 1 & - & - & - & 1 & $\mathrm{R}$ \\
\hline Chromodoris sphoni $i^{1}$ (Marcus, 1971) & - & 2 & 2 & 16 & 1 & 21 & A \\
\hline Glossodoris baumanni $i^{3}$ Bertsch, 1978 & 1 & - & 4 & 2 & - & 7 & SR \\
\hline Glossodoris dalli $^{1}$ (Bergh, 1879) & 5 & 9 & 17 & 2 & - & 33 & A \\
\hline Glossodoris sedna ${ }^{1}$ (Marcus \& Marcus, 1967) & 80 & 24 & 94 & 1 & - & 199 & $\mathrm{~A}$ \\
\hline Hypselodoris agassizii ${ }^{1}$ (Bergh, 1894) & 12 & 42 & 24 & 38 & 2 & 118 & A \\
\hline Mexichromis tura ${ }^{2}$ (Marcus \& Marcus, 1967) & - & - & - & 6 & - & 6 & $\mathrm{R}$ \\
\hline \multicolumn{8}{|l|}{ DENDRODORIDIDAE } \\
\hline Dendrodoris fumata ${ }^{1}$ Rüppell \& Leuckart, 1831 & 1 & 20 & 5 & - & 6 & 32 & A \\
\hline Doriopsilla janaina ${ }^{1}$ Marcus \& Marcus, 1967 & 4 & 9 & 14 & - & 18 & 45 & A \\
\hline $\begin{array}{l}\text { Doriopsilla nigromaculata }{ }^{3} \text { Cockerell en Cockerell \& Eliot, } \\
1905\end{array}$ & 4 & - & 1 & 3 & - & 8 & SR \\
\hline \multicolumn{8}{|l|}{ DOTOIDAE } \\
\hline Doto amyra ${ }^{2}$ (Marcus, 1961) & - & - & 1 & - & - & 1 & $\mathrm{R}$ \\
\hline Doto kya ${ }^{2}$ (Marcus, 1961) & - & 1 & - & - & 1 & 2 & SR \\
\hline Doto lancei $^{2}$ (Marcus \& Marcus, 1967) & - & - & - & - & 6 & 6 & $\mathrm{R}$ \\
\hline Doto sp. $3^{4}$ Marcus, 1961 & - & - & - & - & 44 & 44 & $\mathrm{R}$ \\
\hline \multicolumn{8}{|l|}{ SCYLLAEIDAE } \\
\hline Crosslandia daedali ${ }^{2}$ Poorman \& Mulliner, 1981 & - & - & - & - & 1 & 1 & $\mathrm{R}$ \\
\hline \multicolumn{8}{|l|}{ FLABELLINIDAE } \\
\hline Flabellina bertschi ${ }^{4}$ Gosliner y Kuzirian, 1990 & - & - & - & 122 & - & 122 & $\mathrm{R}$ \\
\hline Flabellina cynara ${ }^{2}$ (Marcus \& Marcus, 1967) & - & - & 1 & - & - & 1 & $\mathrm{R}$ \\
\hline Flabellina telja ${ }^{2}$ (Marcus \& Marcus, 1967) & - & - & 6 & - & - & 6 & $\mathrm{R}$ \\
\hline \multicolumn{8}{|l|}{ EUBRANCHIDAE } \\
\hline Eubranchus sp. ${ }^{2}$ & - & - & - & - & 1 & 1 & $\mathrm{R}$ \\
\hline
\end{tabular}


Tabla 2. Continuación / Continued

\begin{tabular}{|c|c|c|c|c|c|c|c|}
\hline \multicolumn{7}{|l|}{ AEOLIDIIDAE } & \\
\hline Aeolidiella alba ${ }^{1}$ (Risbec, 1928) & 8 & 1 & 14 & 4 & 12 & 39 & A \\
\hline Aeolidiella chromosoma ${ }^{1}$ (Cockerell \& Eliot, 1905). & 90 & 3 & 81 & - & 146 & 320 & A \\
\hline Berghia major $^{2}$ (Eliot, 1903) & 1 & - & - & - & - & 1 & $\mathrm{R}$ \\
\hline Spurilla neapolitana $^{1}$ (Delle Chiaje, 1823 ) & 11 & 1 & 31 & - & 26 & 69 & A \\
\hline \multicolumn{8}{|l|}{ FACELINIDAE } \\
\hline Dondice sp. $1^{2}$ & - & - & - & - & 2 & 2 & $\mathrm{R}$ \\
\hline Favorinus elenalexiae ${ }^{1}$ García \& Troncoso, 2001 & - & 1 & 12 & - & 8 & 21 & SR \\
\hline Phidiana lascrucensis ${ }^{1}$ Bertsch \& Ferreira, 1974 & 69 & 15 & 334 & 38 & 198 & 654 & A \\
\hline Hermosita hakunamatata $^{2}$ (Ortea, Caballer \& Espinosa, 2003) & 1 & - & - & - & - & 1 & $\mathrm{R}$ \\
\hline \multicolumn{8}{|l|}{ TERGIPEDIDAE } \\
\hline Cuthona lagunae $^{2}$ (O' Donoghue, 1926) & 1 & - & - & - & - & 1 & $\mathrm{R}$ \\
\hline Cuthona lizae $^{3}$ Angulo \& Valdés, 2003 & 1 & 2 & 2 & - & - & 5 & SR \\
\hline Total orden & 290 & 138 & 671 & 245 & 576 & 1921 & \\
\hline$S$ (riqueza de especies) & 16 & 17 & 29 & 14 & 27 & & \\
\hline
\end{tabular}

Sitios: $A=$ Tlacopanocha, $B=$ Papagayo, $C=P$. de la Reina, $D=$ Majahua, $E=$ Unicap, $F=$ Disposición espacial local $(A=$ ampliada, $\mathrm{SR}=$ semirestringida, $\mathrm{R}=$ restringida). Ubicación jerárquica $\left({ }^{1}=\right.$ especies dominantes, ${ }^{2}=$ ocasional, ${ }^{3}=$ constantes, ${ }^{4}=$ numerosas poco frecuentes)

Un total de 28 especies son nuevos registros para el litoral del municipio de Acapulco ya que no se encontraron reportes que indicaran registros de estas especies en estas localidades (Aplysia californica, A. vaccaria, Corambe pacifica, Ancula lentiginosa, Atagema alba, Baptodoris mimetica, Doris pickensi, Hoplodoris bramale, Geitodoris heathi, Jorunna tempisquensis, Taringa aivica, Thordisa rubescens, Conualevia alba, C. marcusi, Cadlina flaomaculata, Cadlina sp.1, Chromodoris sphoni, Glossodoris baumanni, G. sedna, Mexichromis tura, Doriopsilla nigromaculata, Doto amyra, D. kya, D. lancei, Berghia major, Cuthona lagunae, Cuthona lizae, Cyerce pavonina) (Figs. 2 y 3).

En los sitios estudiados, el orden mejor representado fue Nudibranchia, el cual se encontró compuesto por 11 familias y 45 especies más una especie de la que sólo se logró identificar hasta nivel de género (Tabla 2). El orden Cephalospidea fue el menos representado y se encontró compuesto por 2 familias y 2 especies (Tabla 1).

La familia Dorididae fue la mejor representada con las especies Baptodoris mimetica, Diaulula aurila, D. greeleyi, Discodoris ketos, Doris granulosa, D. pickensi, Hoplodoris bramale, Geitodoris heathi, Jorunna tempisquensis, Taringa aivica, Thordisa rubescens, seguida por Chromodorididae con 10. El 40,9\% de las familias están representadas por una especie.
Se encontró para los 5 sitios estudiados que 9 especies acumularon el 74,32\% del total de las abundancias, estas son: Phidiana lascrucensis (654), Elysia diomedea (438), Pleurobranchus aerolatus (388), Aeolidiella chromosoma, Glossodoris sedna (199) y Polycera alabe (186), Stylocheilus striatus (125), Flabellina bertschi (122) Hypselodoris agassizii (118).

El orden que se estimó con mayor abundancia relativa en los sitios estudiados fue Nudibranchia (56\%), seguido por Sacoglossa $(19,8 \%)$. Las familias mejor representadas en abundancia relativa fueron: Facelinidae $(19,74 \%)$, Placobranchidae $(12,82 \%)$, Aeolidiidae $(12,50 \%)$ y Pleurobranchidae con $(11,65 \%)$. Seis familias acumularon el $78,21 \%$ de la abundancia total. Cinco familias estuvieron representadas por un solo organismo, estas fueron Bullidae, Scyllaeidae, Goniodoridida, Corambidae y Caliphyllidae. Las especies que se encontraron con mayor abundancia de organismos fueron: Phidiana lascrucensis (654), Elysia diomedea (438), Pleurobranchus aerolatus (388), Glossodoris sedna (199) y Polycera alabe (186).

Para los sitios estudiados se determinó que el 23,44\% de las especies de Opistobranquios identificadas, tienen una disposición espacial local ampliada, entre las especies que presentaron esta característica se encuentran Pleurobranchus aerolatus, Hypselodoris agassizii, Phidania lascrucensis, Aeolidiella alba, A. chromosoma, Navanax aenigamaticus, 
Stylocheilus striatus, Elysia diomedea, Jorunna tempisquensis, Chromodoris sphoni, Glossodoris dalli, G. sedna, Dendrodoris fumata, Doriopsilla janaina y Spurilla neapolitana. Con disposición espacial local semirestringida se encontró al 32,8\% de las especies y el 43,75\% del total de las especies identificadas se determinó con disposición restringida (Tabla 1).

En cada uno de los sitios el orden Nudibranchia fue el mejor representado en especies. En Tlacopanocha el orden Anaspidea se presentó como el segundo lugar en representación por especies. En el sitio Papagayo el segundo lugar fue ocupado por el orden Sacoglossa. En los sitios Parque de la Reina, Majahua y Unicap el segundo lugar lo presentó el orden Notaspidea.
Del total de familias identificadas para los sitios estudiados, el lugar donde se encontró una mayor representación de familias fue en Parque de la Reina (15). El sitio con menor representación fue Tlacopanocha (10).

La mayor riqueza de especies fue encontrada en Parque de la Reina con 41 especies y la menor en Majahua, con 20 especies.

En los sitios Tlacopanocha, Parque de la Reina, Majahua y Unicap el orden Nudibranchia se presentó como el de mayor abundancia relativa. En Morro del Papagayo el orden que presentó la mayor abundancia relativa fue el Sacoglossa.

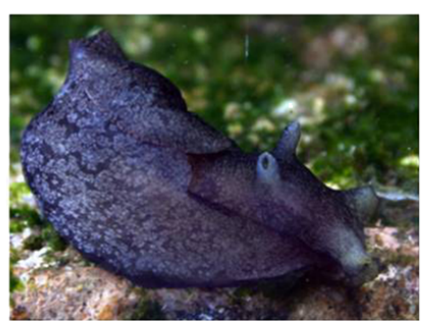

Aplysia vaccaria

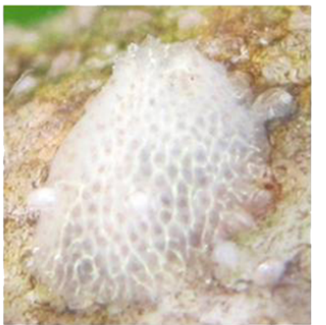

Corambe pacifica

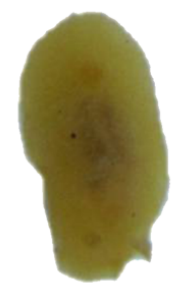

Baptodoris mimetica

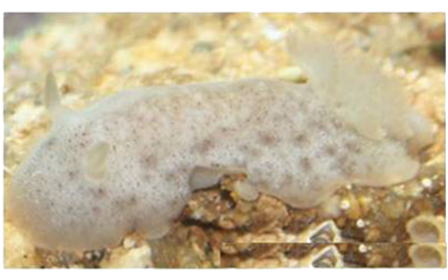

Doris pickensi

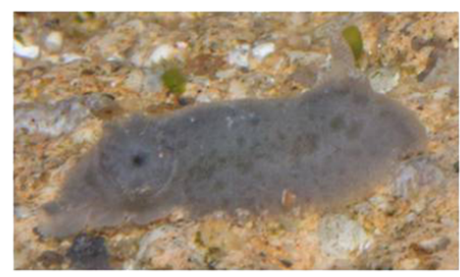

Jorunna tempisquensis

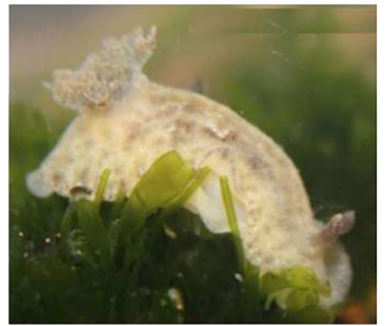

Taringa aivica

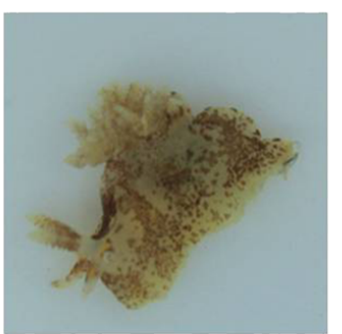

Ancula lentiginosa

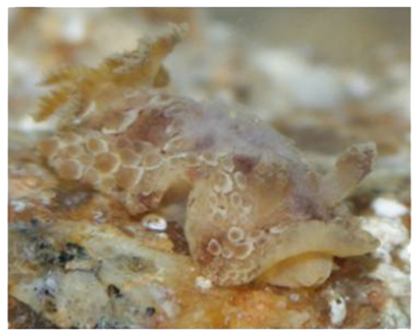

Hoplodoris bramale

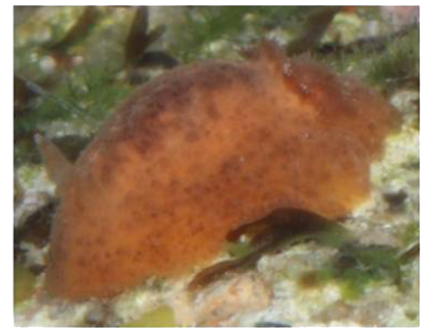

Thordisa rubescens

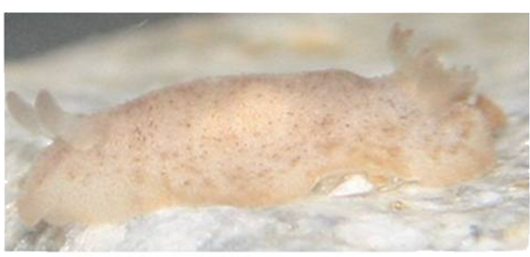

Atagema alba

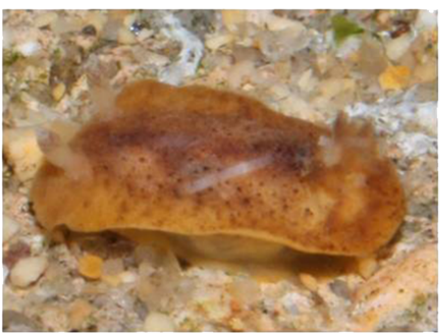

Geitodoris heathi

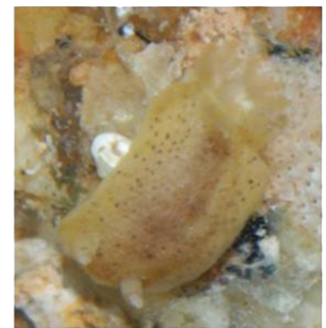

Doriopsilla nigromaculata

Figura 2. Nuevos registros de distribución de especies de la Subclase Opisthobranchia (Mollusca: Gastropoda), para Acapulco, México / New distribution records of species, Subclass Opisthobranchia (Mollusca: Gastropoda), to Acapulco, Mexico 
El orden Anaspidea se halló como el segundo en abundancia en Tlacopanocha y Papagayo, el orden Sacoglossa en Parque de la Reina y Majahua y el orden Notaspidea en Unicap.

El orden Cephalaspidea se encontró como el de menor abundancia en Tlacopanocha, Parque de la Reina, Morro del Papagayo y Unicap. En Majahua el orden Anaspidea se presentó como el de menor abundancia.

En Tlacopanocha la familia mejor representada en abundancia fue Aplysiidae, en Papagayo fue Placobranchidae, en Parque de la Reina, fue Facelinidae, en Majahua fue Flabellinidae y en Unicap Pleurobranchidae.

Unicap, fue el sitio donde se encontró la mayor abundancia relativa $(31,65 \%)$, seguido por Parque de la Reina $(26,26 \%)$, Papagayo $(17,49 \%)$, Tlacopanocha $(15,36 \%)$ y Majahua $(9,29 \%)$.
En Tlacopanocha las especies más abundantes fueron Aeolidiella chromosoma (90), Aplysia juliana (85), G. sedna (80), Phidiana lascrucensis (69), Elysia diomedea (54).

En el sitio Papagayo se encontró con mayor abundancia a Elysia diomedea (200), Stylocheilus striatus (116), Hoffmanola hansi (53) e Hypselodoris agassizii (42).

En el sitio Parque de la Reina las especies más abundantes fueron Phidiana lascrucensis (334), Elysia diomedea (132), G. sedna (94), Aeolidella chromosoma (81).

En el sitio Majahua se encontraron con mayor abundancia a Flabellina bertschi (122), Elysia diomedea (52), Hypselodoris agassizii (38).

En el sitio Unicap, se encontró con mayor abundancia a Aeolidiella chromosoma (146), Phidania lascrucensis (198), Polycera alabe (186) y Pleurobranchus aerolatus (318).

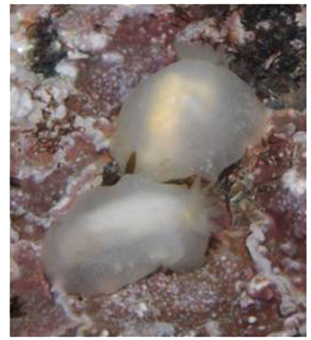

Conualevia alba

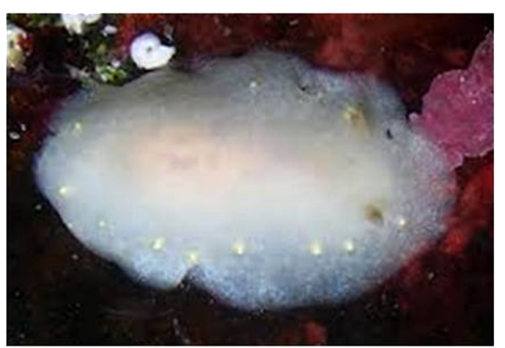

Cadlina sp.1

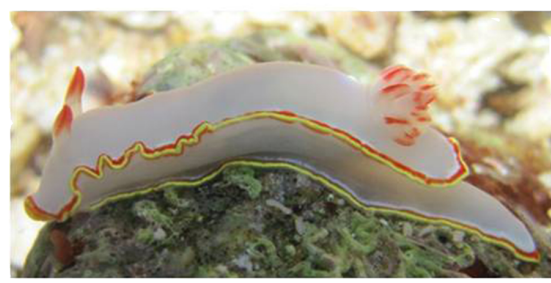

Glossodoris sedna

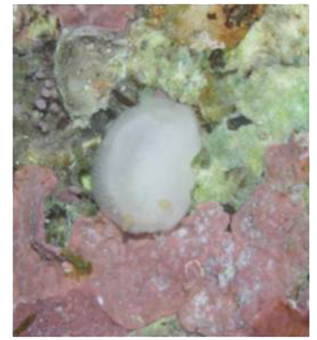

Conualevia marcusi

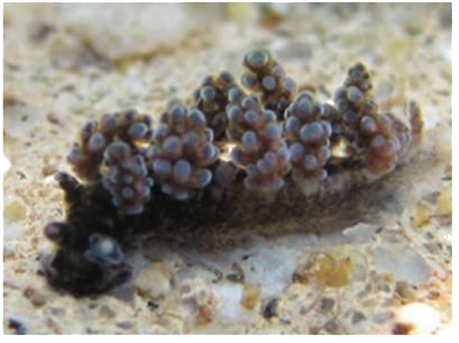

Doto lancei

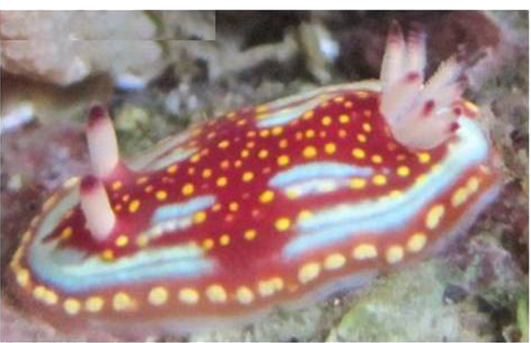

Chromodoris sphoni

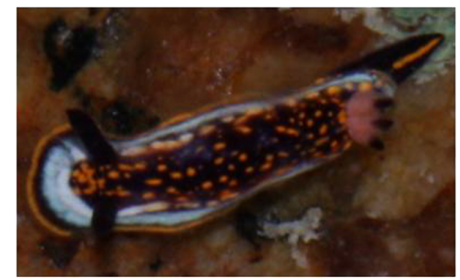

Mexichromis tura

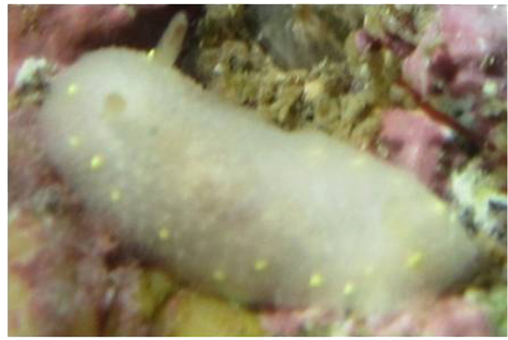

Cadlina flavomaculata

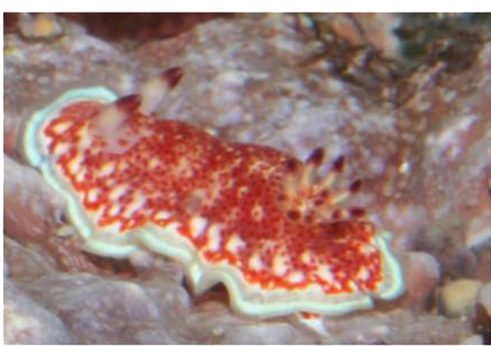

Glossodoris baumanni

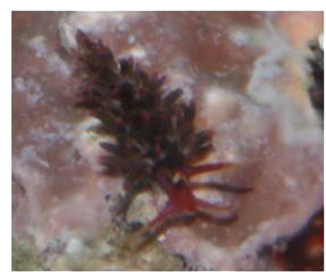

Cuthona lagunae

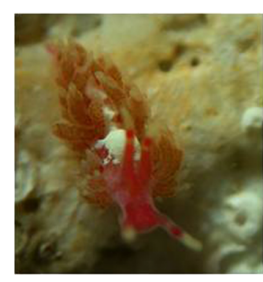

Cuthona lizae

Figura 3. Nuevos registros de distribución de especies de la Subclase Opisthobranchia (M ollusca: Gastropoda), para Acapulco, México / New distribution records of species, Subclass Opisthobranchia (Mollusca: Gastropoda), to Acapulco, Mexico 
El sitio Unicap, presentó el mayor número de especies con disposición espacial local restringida (13), seguido por Parque de la Reina (6), Tlacopanocha (4), Majahua (3) y Papagayo (2). Hoffmanola hansi y Polycera alabe se presentaron únicamente en el sitio Unicap y fueron abundantes.

Con base en grado de aparición de las especies dentro de la comunidad, se encontró que las especies dominantes representan el 28,78\% (Tablas 1 y 2), son numerosas poco frecuentes el $15,16 \%$, ocasionales el $48,48 \%$ y constantes $7,58 \%$.

Se observó que la mayor similitud en cuanto a las abundancias de opistobranquios, correspondió a los sitios Tlacopanocha y Parque de la Reina. En un segundo nivel de relación, se registró que el primer grupo formado presentó similitud conjunta con el sitio Papagayo. Los sitios Majahua y Unicap, presentaron la mayor disimilitud (Fig. 4).

\section{Discusión}

El presente trabajo adiciona a la lista de la Subclase Opisthobranchia a 28 especies. Estos nuevos registros que se reportan para Acapulco, indican la necesidad de avanzar con el conocimiento de este grupo en la ecoregión del Pacífico Transicional Mexicano.

Los ordenes de la Subclase Opisthobranchia encontrados en Acapulco, son los mismos que fueron documentados para Colima, Michoacán y Guerrero (Hermosillo \& Behrens 2005); para Jalisco (Hermosillo 2006, Hermosillo \& Gosliner 2008); para Panamá (Hermosillo 2004). Esto sugiere que los 5 ordenes que forman la Sublcase se distribuyen de igual modo en las Ecoregiones del Pacífico Transicional Mexicano y en la del Pacífico Centroamericano. El orden Nudibranchia, es el mejor representado, tanto en riqueza de especies como en abundancia en los 5 sitios estudiados.

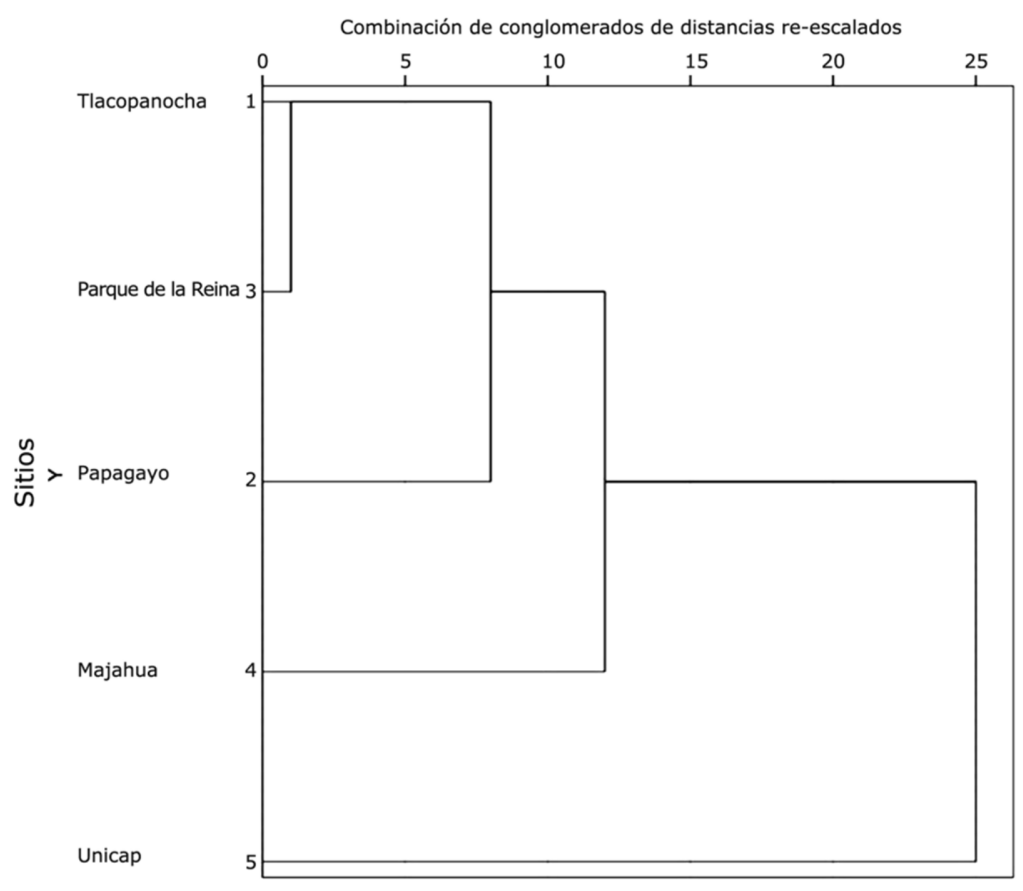

Figura 4. Similitud entre los valores de las abundancias de la Subclase Opisthobranchia (M ollusca: Gastropoda), registradas en 5 sitios del litoral de Acapulco / Analysis of similarity between the values of the abundances of Subclass Opisthobranchia (Mollusca: Gastropoda), recorded at 5 sites of the coast to Acapulco 
Las familias del orden Nudibranchia fueron los mejor representados en riqueza de especies (Montoya et al. 2001 ${ }^{1}$, Hermosillo 2004, 2006 y Angulo 2005), lo que concuerda con los resultados encontrados para Acapulco.

Hermosillo \& Behrens (2005), encontraron que la representación por familias del orden Nudibranchia es muy parecida a la encontrada en Acapulco por el este trabajo, no obstante, ellos reportaron mayor cantidad de familias. Esta diferencia se puede atribuir a que ellos muestrearon en un área geográfica mayor que comprendió los estados de Colima, Michoacán y Guerrero.

Hermosillo (2004), en Isla Coiba, Panamá encontró que Chromodorididae y Polyceridae fueron las familias mejor representadas. Hermosillo (2006), para Bahía Banderas, que se sitúa dentro de la ecorregión Pacífico Transicional Mexicano, encontró que las familias mejor representadas en riqueza de especies fueron Dorididae y Chromodorididae. Castillo (2010), en Mazatlán, encontró como la familia más representativa a Aplysiidae. Este trabajo encuentra el mismo resultado reportado para Bahía Banderas (Hermosillo 2006).

El 46\% de las especies encontradas por este trabajo son coincidentes con las reportadas para los estados de Colima, Michoacán y Guerrero (Hermosillo \& Behrens 2005), y el 36\% con las reportadas para Jalisco (Hermosillo 2006).

La alta riqueza de especies encontrada en bahía Banderas, Jalisco (140) (Hermosillo 2006), para el Golfo de California (117) (Angulo 2005), en la Bahía de los Ángeles, Golfo de California, Mar de Cortés (81) (Bertsch 2008), para Puerto Manzanillo, Colima, Puerto Lázaro Cárdenas, Michoacán y Zihuatanejo, Guerrero (76) (Hermosillo \& Behrens 2005), para Parque Nacional Coiba, Panamá (89) (Hermosillo \& CamachoGarcía 2006), parece estar relacionada con sitios ubicados dentro de bahías o en el Mar de Cortés que presentan en común aguas con intensidad de oleaje bajo. Por el contrario, estudios realizados en costas abiertas, con oleaje intenso, presentaron menor riqueza de especies, como los estudios realizados para California (32) (Bertsch et al. 1972), en el Archipielago de Revillagigedo (37) (Hermosillo \& Gosliner 2008), para la costa norte de Perú (17) (Nakamura 2007). Spight (1976), encontró que la distribución de las especies varía con el grado de exposición al oleaje, el tipo de sustrato o ambos factores.

El valor de la riqueza de especies encontrada en los sitios estudiados por el presente trabajo es de las más altas que se han reportado para el Pacífico mexicano. Un aspecto que puede estar influyendo en la riqueza de especies en Acapulco es la heterogeneidad del hábitat con diversos relieves y rugosidad de sustratos o tipos de microhábitat, donde las especies pueden encontrar refugio y alimentación. Levinton (1982), señala que un hábitat más heterogéneo, con muchos recursos y muchos tipos de sustrato soporta muchas especies.

La mayor riqueza de especies se encontró en Parque de la Reina, el sustrato en este sitio es muy heterogéneo, que va desde la acumulación artificial de rocas de tamaños variables, hasta restos de pisos y columnas de concreto y la intensidad del oleaje es medio. Esta heterogeneidad del sustrato permite el establecimiento de una mayor variedad de especies. El sitio Unicap, constituido por sustrato artificial suspendido en la columna de agua y protegido del impacto directo de las olas ocupó el segundo lugar en riqueza de especies.

Los sitios que presentaron menor heterogeneidad del sustrato, como Majahua caracterizada por cantos rodados en procesos de abrasión, cuenta con bloques de textura lisa al tacto y poca presencia de fisuras y/o hendiduras, se observaron con menor riqueza de especies. Flores-Rodríguez et al. (2012) en una investigación llevada a cabo en 9 sitios distribuidos en la costa del estado de Guerrero encontraron que la riqueza de especies de moluscos, está asociada a la estabilidad del hábitat y también a la intensidad del oleaje de cada sitio, de tal manera que sitios más inestables, que son aquellos cuyo sustrato está constituido por cantos rodados y con menor grado de intensidad del oleaje, presentaron mayor riqueza, mientras que sitios con sustrato más estable, conformados por grandes bloques fijos con pocas fisuras y grietas así como una alta intensidad del oleaje presentaron menor riqueza de especies.

De acuerdo con los reportes para el Golfo de California (Angulo 2000), y de Bahía Banderas, Jalisco(Hermosillo 2006), el orden Nudibranchia es el mejor representado en abundancia, un resultado similar se encontró para Acapulco por esta investigación.

Con respecto a las abundancias por familias, no se encontraron reportes que organizaran la información con base en este taxón, sin embargo, en el trabajo de Hermosillo (2006), se observa que la familia mejor representada en abundancia fue Flabellinidae, seguida de Chromodoridae. Esta investigación no coincide con el reporte anterior.

Se ha reportado, por diferentes autores la abundancia relativa que acumulan 9 especies de opistobranquios, para California 87\% (Nybakken 1978), para el Golfo de California, 67,65\% (Angulo 2003), para el Golfo de California $82 \%$ (Bertsch 2008), para bahía Banderas, Jalisco 49,8\% (Hermosillo 2006). Un resultado similar se encontró para Acapulco donde 9 especies acumularon el 74,32\% de la abundancia total.

Angulo (2003) encontró en el Golfo de California a Elysia diomedea entre las especies más abundantes y Hermosillo (2006) para Jalisco, reportó a Glossodoris sedna. Estas dos 
especies coinciden con las reportadas por este trabajo con mayor abundancia. Hermosillo et al. (2006), mencionan que muchas especies van y vienen de hábitats locales, despareciendo por varios años en donde alguna vez fueron abundantes, y después apareciendo de nuevo.

La mayor abundancia se presentó en el sitio Unicap, y que a diferencia de los otros sitios, se encuentra en un ambiente pelágico, fuera de la zona intermareal, la combinación del ambiente pelágico así como la presencia de hendiduras distribuidas uniformemente en las canastas suspendidas, ofrecen un espacio continuo habitable, lo que se expresó en la mayor abundancia de organismos.

La menor abundancia se presentó en el sitio Majahua, y aunque también se encuentra en una bahía con intensidad de oleaje bajo, tiene sustratos menos heterogéneos, formado por grandes bloques de textura lisa al tacto y poca presencia de hendiduras, canales y rocas sueltas lo que reduce el número de espacios habitables para el grupo de opistobranquios.

El sitio Unicap presentó la mayor proporción de especies con disposición espacial local restringida (13), esto puede deberse a su ubicación en un ambiente pelágico donde recibe oleaje de baja intensidad, que de entre los sitios muestreados, es el único fuera de la zona intermareal rocosa. El resto de los sitios, que se ubican en ambiente intermareal, presentaron en común un menor número de especies con disposición local restringida.

Se encontraron especies abundantes y con disposición espacial local restringida, como Flabellina bertschi en Majahua y Hoffmanola hansi en Papagayo. La alta abundancia de estas especies son un indicador de una adaptación especializada a las condiciones ambientales de los sitios en que se les encontró.

Para Acapulco, con base en la abundancia relativa y frecuencia de ocurrencia se encontró como especies dominantes en la comunidad a Navanax aenigmaticus, Aplysia parvula, Stylocheilus striatus, Pleurobranchus aerolatus, Oxynoe panamensis, Elysia diomedea, Diaulula greeleyi, Jorunna tempisquensis, Chromodoris sphoni, Glossodoris dalli, Glossodoris sedna, Hypselodoris agassizii, Dendrodoris fumata, Doriopsilla janaina, Aeolidiella alba, Aeolidiella chromosoma, Spurilla neapolitana, Favorinus elenalexiae, Phidiana lascrucensis.

A partir de registrar que la mayor similitud en abundancias correspondió al par de sitios conformado por Tlacopanocha y Parque de la Reina, así como los grupos con menor similitud dado los valores extremos fueron Majahua y Unicap, respectivamente, se observaron características del hábitat que son comunes entre los sitios, y que pueden estar contribuyendo en la distribución de las abundancias de las especies.
Los sitios Tlacopanocha y Parque de la Reina, que presentan característica en común, sustratos heterogéneos formado por escombros, pedazos de concreto, rocas de tipo granitogranodiorita y rocas metamórficas, que varían mucho en cuanto a sus dimensiones, donde es posible encontrar desde grava hasta grandes bloques, la superficie del sustrato es áspero al tacto. También tienen en común que las olas no chocan directamente contra el sustrato, por lo que pueden considerarse sitios protegidos.

En segundo lugar, de similitud en abundancia fue formado este primer subgrupo y el sitio Papagayo, que es un islote que presenta al igual que el primer subgrupo, un sustrato heterogéneo, con superficies ásperas y en el interior se forman pozas de marea que presentan un sustrato arenoso con restos de conchas, y rocas sueltas. Este sitio, por encontrarse frente a la bocana, recibe oleaje de intensidad media a baja, por lo que también puede considerarse sitio protegido.

\section{Agradecimientos}

El agradecimiento de los autores al Dr. Ángel Valdés por su valiosa colaboración en corroborar la identificación de la mayoría de las especies, y también a los evaluadores anónimos que con sus sugerencias permitieron mejorar este manuscrito.

\section{LITERATURA CITADA}

Angulo CO. 2000. Moluscos Opistobranquios (Mollusca: Opistobranchiata) de Baja California Sur, México. Tesis de Licenciatura, Departamento de Biología Marina, Universidad Autónoma de Baja California Sur, La Paz, 176 pp.

Angulo CO. 2003. Variación espacio-temporal de las poblaciones de Opistobranquios (Mollusca: Opisthobranchia) en tres localidades de B.C.S. México. Tesis de Maestría. Instituto Politécnico Nacional, Centro Interdisciplinario de Ciencias Marinas, La Paz, Baja California Sur, México, 64 pp.

Angulo CO. 2005. A four year survey of the opistobranch fauna (Gastropoda, Opistobranchia) from Baja california Sur, México. Vita Malacológica 3: 43-50.

Arriaga L, E Vázquez, J González, R Jiménez, E Muñoz \& V Aguilar. 1988. Regiones prioritarias marinas de México. Comisión Nacional para el Conocimiento y Uso de la Biodiversidad, México, D. F. <http://www.conabio.gob.mx/ conocimiento/regionalizacion/doctos/marinas.html>

Ávila-Poveda OH, QY Abadia-Chanona, R HerreraFragoso \& B Dayrat. 2014. Review of the geographic distribution of Hoffmannola hansi (Gastropoda: Pulmonata) in the Mexican Pacific. Revista Mexicana de Biodiversidad 85(2): 463-471. 
Behrens DW \& A Hermosillo. 2005. Eastern Pacific Nudibranchs: a guide to the Opisthobranchs from Alaska to Central America, 137 pp. Sea Challengers, Monterey.

Behrens DW, TM Gosliner \& A Hermosillo. 2009. A new species of dorid nudibranch (Mollusca) from the Revillagigedo Islands of the Mexican Pacific. Proceedings of the California Academy of Sciences 60 (11): 423-429.

Bertsch H. 1995. A three-year phonological analysis of subtidal populations of Opisthobranch Mollusks at Bahía de Los Angeles, Baja California, México. Proceedings of the Pacific Division of the American Association for the Advancement of Science, 76th Annual Meeting 14(1): 34.

Bertsch H. 2008. Opistobranquios. En: Danemann G \& E Ezcurra (eds). Bahía de los Ángeles: Recursos naturales y comunidad. Línea base 2007, pp. 319-338. Secretaría del Medio Ambiente y Recursos Naturales, Instituto Nacional de Ecología, Pronatura Noroeste A.C, San Diego Natural History Museum, México.

Bertsch H. 2009. Biogeography of northeast Pacific opisthobranchs from Point Conception, California, USA, to the Galápagos Islands, Ecuador: Comparative faunal province studies of the Sea of Cortez. Western Society of Malacologists, Annual Report 40: 32-34.

Bertsch H, TM Gosliner, R Wharton \& G Williams. 1972. Natural history and occurrence of Opistobranch gastropods from the open coast of San Mateo Country, California. Veliger 14(3): 302-312.

Camacho-García YE \& TM Gosliner. 2008. Systematic revision of Jorunna Bergh, 1876 (Nudibranchia: Discodorididae) with a morphological phylogenetic analysis. Journal of Molluscan Studies 74: 143-181.

Carmona L, BR Lei, M Pola, TM Gosliner, A Valdés \& JL Cervera. 2014. Untangling the Spurilla neapolitana (Delle Chiaje, 1841) species complex: A review of the genus Spurilla Bergh, 1864 (Mollusca: Nudibranchia: Aeolidiidae). Zoological Journal of the Linnean Society 170: 132-154. <doi:10.1111/zoj.12098>

Carranza-Edwards A, M Gutiérrez-Estrada \& R RodríguezTorres. 1975. Unidades morfo-tectónicas continentales de las Costas Mexicanas. Anales del Instituto de Ciencias Del Mar y Limnología, UNAM, México 2(1): 81-88.

Castillo FC. 2010. Inventario de bivalvos y opistobranquios (Phyllum Mollusca) del estero de Urías y costas de Mazatlán Sinaloa, México. Tesis de Biólogo Pesquero, Universidad Autónoma de Sinaloa, Mazatlán, 119 pp.

De La Lanza EG. 1991. Oceanografía de los mares mexicanos, 569 pp. AGT Editor, México.

Flores-Rodríguez P, R Flores-Garza, S García-Ibáñez, A Valdés-González, J Violante-González, E SantiagoCortés, L Galeana-Rebolledo, C Torreblanca-Ramírez. 2012. Mollusk species richness on the rocky shores of the state of Guerrero, Mexico, as affected by rains and their geographical distribution. Natural Resources 3: 248-260.
Flores-Rodríguez P, R Flores-Garza, S García-Ibáñez, C Torreblanca-Ramírez, L Galeana-Rebolledo \& E Santiago-Cortes. 2014. Mollusks of the Rocky Intertidal Zone at Three Sites in Oaxaca, Mexico. Open Journal of Marine Science 4: 326-337. <http://dx.doi.org/10.4236/ ojms.2014.44029>

Ginsburg DW \& VJ Paul. 2001. Chemical defenses in the sea hare Aplysia parvula: importance of diet and sequestration of algal secondary metabolites. Marine Ecology Progress Series 215: 261-274.

Goddard JHR. 2005. Ametamorphic direct development in Dendrodoris behrensi (Nudibranchia: Dendrodorididae), with a review of developmental mode in the family. Proceedings of the California Academy of Sciences 56(19): 201-211.

Goddard JHR \& A Valdés. 2015. Reviving a cold case: two northeastern Pacific dendrodorid nudibranchs reassessed (Gastropoda: Opisthobranchia). The Nautilus 129: 31-42.

Gosliner TM. 1991. The opisthobranch gastropod fauna of the Galápagos Islands. In: James MJ (ed). Galápagos marine invertebrates: taxonomy, biogeography, and evolution in Darwin's islands. Topics in Geobiology 8: 281-305. Plenum, New York.

Guido C \& MT Ghiselin. 1998. Chemical defense and evolution in the Sacoglossa (Mollusca: Gastropoda: Opisthobranchia) Chemoecology 8: 51-60.

Hermosillo A. 2003. New distributional records of Opistobranch mollusks for Bahía de Banderas, México (Tropical Eastern Pacific). The Festivus 35(3): 21-28.

Hermosillo A. 2004. Opistobrach mollusks of Parque Nacional of Coiba, Panama (Tropical Eastern Pacific). The Festivus 36(9): 105-117.

Hermosillo A. 2006. Ecología de los Opistobranquios de Bahía de Banderas, Jalisco-Nayarit, México. Tesis de Doctorado, Centro Universitario de Ciencias Biológicas y Agropecuarias, Universidad de Guadalajara, México, 151 pp.

Hermosillo A \& D Behrens. 2005. The Opistobranchs fauna (Gastropoda, Opistobranchia) of the México States of Colima, Michoacán and Guerrero: filling in the faunal gap. Vita Malacológica 3: 11-22.

Hermosillo A \& YE Camacho-García. 2006. A note on the Opisthobranchs of Parque Nacional de Coiba, Panamá (Tropical Eastern Pacific). The Festivus 38(9): 95-98.

Hermosillo A \& TM Gosliner. 2008. The Opistobranch fauna of the Revillagigedo Archipelago, Mexican Pacific. The Festivus 40(2): 25-34.

Hermosillo A \& A Valdés. 2004. Two new species of dorid nudibranchs (Mollusca, Opisthobranchia) from Bahía de Banderas and La Paz, Mexico. Proceedings of the California Academy of Sciences 55(28): 550-560.

Hermosillo A \& A Valdés. 2007a. Five new species of aeolid nudibranchs (Mollusca, Opisthobranchia) from the tropical eastern Pacific. American Malacological Bulletin 22: 119137. <doi:10.4003/0740-2783-22.1.119> 
Hermosillo A \& A Valdés. 2007b. A new Polycera (Opisthobranchia: Mollusca) from Bahía de Banderas, México. Proceedings of the California Academy of Sciences 58: 477-484.

Hermosillo A \& A Valdés. 2008. Two new species of opisthobranch mollusks from the tropical eastern Pacific. Proceedings of the California Academy of Sciences 59:521532.

Hermosillo A, DW Behrens \& E Ríos-Jara. 2006. Opistobranquios de México: Guía de babosas marinas del Pacífico, Golfo de California y las islas oceánicas, 143 pp. CONABIO, México.

Keen AM. 1971. Sea shells of Tropical West America, 1064 pp. Stanford University Press, California.

Levinton JS. 1982. Marine ecology, 526 pp. Prentice-Hall, Englewood Cliffs.

Millen S. 2006. A new species of Adalaria (Nudibranchia: Onchidorididae) from the Northeastern Pacific. Proceedings of the California Academy of Sciences 57(8): 357-364.

Millen S \& A Hermosillo. 2007. The genus Flabellina Voight, 1834 (Mollusca: Opistobranchia) from Bahía de Banderas (Pacific Coast of Mexico) with ecological observations, the description of a new species and the redescription of Flabellina cynara. Proceeding of the California Academy of Sciences 58(26): 543-559.

Millen SV \& TM Gosliner. 1985. Four new species of dorid nudibranchs belonging to the genus Aldisa (Mollusca: Opisthobranchia), with a revision of the genus. Zoological Journal of the Linnean Society 84: 195-233.

Nakamura K. 2007. Especies bentónicas de Opistobranchia (Mollusca: Gastropoda) presentes en el litoral del norte peruano. Revista de Biología Peruana, Número Especial 13(3): 255-257.

Nybakken J. 1978. Abundance, diversity and variability in a California intertidal nudibranch assemblage. Marine Biology 45(2): 129-146.
Pola M, V Padulab, TM Gosliner \& JL Cerverad. 2014. Going further on an intricate and challenging group of nudibranchs: description of five novel species and a more complete molecular phylogeny of the subfamily Nembrothinae (Polyceridae). Cladistics 30(6): 607-634.

Shipman C \& TM Gosliner. 2015. Molecular and morphological systematics of Doto oken, 1851 (Gastropoda:Heterobranchia), with descriptions of five new species and a new genus. Zootaxa 3973(1): 57-101. <doi.org/ 10.11646/zootaxa.3973.1.2>

Sokal RR \& FJ Rohlf. 1969. Biometry. The principles and practices of statistics in biological research, 587 pp. W. H. Freeman, San Francisco.

Spight TM. 1976. Censuses of rocky shore prosobranchs from Washington and Costa Rica. The Veliger 18(3): 309-317.

Valdés Á. 2004. Phylogeography and phyloecology of dorid nudibranchs (Mollusca, Gastropoda). Biological Journal of the Linnean Society 83(4): 551-559.

Valdés Á \& O Angulo-Campillo. 2004. Systematics of pelagic aeolid nudibranchs of the family Glaucidae (Mollusca, Gastropoda). Bulletin of Marine Science 75(3): 381-389.

Wagele H \& RC Willan. 2000. Phylogeny of the Nudibranchia. Zoological Journal of the Linnean Society 130(1): 83-181.

Wilkinson T, E Wiken, J Bezauri-Creel, T Hourigan, T Agardy, H Herrmann, L Janishevski, C Madden, L Morgan \& M Padilla. 2009. Ecorregiones marinas de América del Norte, 197 pp. Comisión para la Cooperación Ambiental, Montreal. <http://www.cec.org/islandora/en/item/ 3256-marine-ecoregions-north-america-es.pdf $>$

Zamora-Silva A \& E Naranjo-García. 2008. Los Opistobranquios de la colección nacional de moluscos. Revista Mexicana de Biodiversidad 79: 333-342. 